\title{
On the Creation of a New Diagnostic Model for Fetal Well-Being on the Base of Wavelet Analysis of Cardiotocograms
}

\author{
Carlo Cattani · Olga Doubrovina · Sergei Rogosin • \\ Sergei L. Voskresensky • Elena Zelianko
}

Received: 25 July 2006 / Accepted: 7 August 2006 / Published online: 14 September 2006

(C) Springer Science+Business Media, Inc. 2006

\begin{abstract}
The article is devoted to the description of the results of wavelet analysis of fetal heart rate detecting by cardiotocography method. A number of conclusions are made on the base of such an analysis.

It is a part of the research program of creation of a new diagnostic model estimating fetal conditions in antepartum period.
\end{abstract}

Keywords Fetal heart - Wavelets · Cardiotocography · Antepartum

\section{Introduction}

During pregnancy and labour, the medical staffs employ fetal heart rate (FHR) recordings for evaluation of fetal well being. Antepartum FHR monitoring in the form of the nonstress test (NST) by using cardiotocography (CTG) is popular because

C. Cattani $(\bowtie)$

DiFarma, Universita di Salerno,

I-84084, Via Ponte Don Melillo, Fisciano (SA), Italy

e-mail: ccattani@unisa.it

O. Doubrovina

Belarusian National Technical University,

220027, 65, Nezavisimosti ave, Minsk, Belarus

e-mail: dubrovina@bsu.by

S. Rogosin

Belarusian State University,

220050, 4, Nezavisimosti ave, Minsk, Belarus

e-mail: rogosin@bsu.by

S. L. Voskresensky · E. Zelianko

Belarusian Medical Academy of Postgraduate Education,

220714, 3, P. Brovka str., Minsk, Belarus

e-mail: zelenko_elena@rambler.ru of (1) it is easier to use; (2) it has no contra-indications; (3) it is non-invasive; (4) may be used repeatedly; (5) it can be applied in the outpatient department [1]. Necessity of visual assessment by physicians causes poor intra-observer and inter-observer reliability. The interpretation of observed CTGs is a skill which is acquired through years of experience [2].

The normal FHR pattern is characterized by a baseline frequency between 110 and 160 beats per minute, presence of periodic accelerations (FHR-increasing more than 15 beats/min lasting more than $15 \mathrm{~s}$ ), normal heart rate variability with a bandwidth between 5 and 25 beats per minute (it is called reactive pattern of NST reading) and the absence of decelerations (FHR-decreasing more than 15 beats/min lasting more than $15 \mathrm{~s}$ ) [1]. The poor fetal condition is associated with decreasing of FHR variability. However, decreasing FHR can be also observed in normal fetal condition during such fetal behavioral state as so called "quiet sleep" (which can last for about 40 minutes). This FHR pattern without accelerations with a low FHR variation is called "nonreactive" result of NST. A diagnosis of fetal distress should not be determined on the basis of nonreactive pattern of NST if the recording did not last more than 45 minutes. There is no criteria to distinguish nonreactive pattern of NST during quiet sleep of normal fetus from FHR pattern in poor fetal condition [3].

If obstetricians conclude from CTG that FHR pattern is normal then it means almost surely the good condition of a fetus. It makes the cardiotocography' method useful. However, in cases of FHR patterns considered "abnormal" by the obstetricians, at least half of these turn out to have been false alarm. Thus the number of operative interventions is dramatically increased since the introduction of CTG; many of these having been unnecessary [4]. 
Automatic analysis is created to overcome this difficulty. Computerized CTG analysis can lead to uniform and objective interpretation by providing a reproducible determination of FHR characteristics. Computerized evaluation of FHR also allows to estimate those characteristics that cannot be assessed by visual analysis. For example-short-term variation (STV, criteria Dawes-Redman) — pulse interval differences for 3.75-s epoch, which is applied in the most widely used system for the numerical analysis of NST, namely, in the computer program made by Sonicaid System. Computerized analysis allows us to assess FHR variability more exactly and to raise accuracy of perinatal outcome prediction. Presence of episodes of a high variation lasting 10 or more minutes indicates more than $99 \%$ of normal NST readings. However, using this program it is not possible to assess fetal well-being during quiet sleep because the low FHR variation of healthy fetuses during quiet sleep cannot be distinguished from the low variation of compromised fetuses [5].

Recently, it is developed the heart rate variability (HRV) assessment in the frequency domain using digital signal processing techniques. Power spectral descriptions of HRV (different kind of Fourier analysis) are now widely used tools for the assessment of autonomic function and the prediction of outcome in some kinds of pathological conditions [6]. Wavelet transform, on the other hand, is a very new analysis method. The main advantage of wavelet transform analysis is in the obtaining of the additional information about the period of time when the various spectral components appear in the signal. In this way, sudden amplitude and/or frequency jumps can be detected. Using wavelet transform analysis of heart rate variability in adults has demonstrated more possibility in prediction of outcome in some kinds of pathological conditions [7]. Wavelet transform has been applied only in a few studies for the analysis of FHR during labour [8]. The aim of our work is to study possibility of analysis of amplitude-frequency characteristics FHR which is obtained by using wavelet transform analysis for indication of fetal condition. We determine certain amplitude-frequency characterization of the FHR in a number of frequency bands (see [9]). By using multiply regression method we propose a new diagnostic method for prediction of the anterpartum asphyxia. The diagnostic efficiency of the model is analyzed on the base of such analysis.

\section{Methods}

We have analyzed 121 FHR records. 37 FHR records were obtained in 9 cases of intrauterine deaths from asphyxia during antepartum period (during pregnancy) from 1992 through 2003 in Obstetric Clinic of Minsk District and Clinical Hospital No. 7 in Minsk. The fetuses of these women had pathoanatomical sigh of fetal asphyxia. 84 FHR records were obtained from 31 women with normal perinatal outcome.

The CTGs were registered during pregnancy using external method by the cardiotocograph "Corometrics." The mean duration of records was $18 \mathrm{~min}\left(\mathrm{CI}_{95 \%}=17-19 \mathrm{~min}\right)$. The CTGs with purely sinusoidal shape were excluded from analysis since they surely means a bad situation with a fetal (see, e.g. [1]). The sampling frequency cardiotocography "Corometrics" was $1 \mathrm{~Hz}$.

To develop amplitude-frequency characterization of the FHR we use wavelet analysis. The original CTG's have a a form of a continuous curve of a finite length. Anyway, the real information is presented only in a collection of points on the curve. Therefore, starting from integral (continuous) wavelet transform we deal later only with discrete information which is analyzed by using discrete wavelet transform. To do this we create an algorithm for such a discrete analysis. Let us briefly describe it.

The CTGs are converted in digital form. Before using wavelet analysis digital data of CTGs we transfer in form which was possible for wavelet analysis. First, the characteristic points of the CTGs are obtained. Then we detect a new signal length which was equal the second power. For this we applied the following formula:

$N=2^{\left[\log _{2} n\right]+1}$

We rebuilt the data collection into time series by exploiting extrapolation/interpolation and possible moving of the above characteristic points.
Fig. 1 Fetal heart rate recording

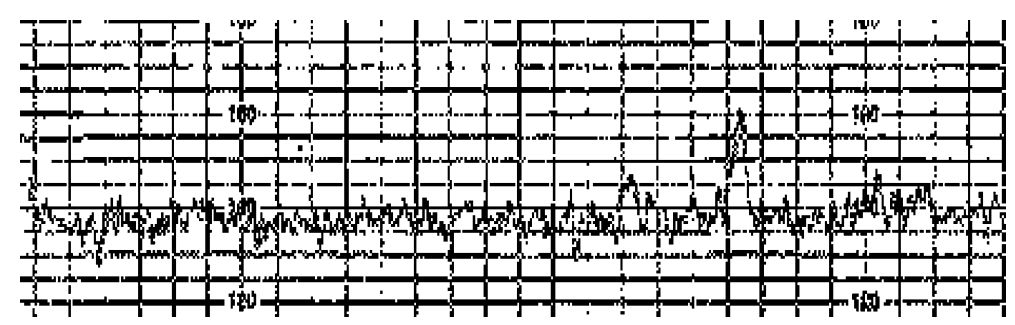


For computing signal value $x\left(t_{i}\right)$ in new data set $t_{i}$ we apply polynomial interpolation in internal range or extrapolation out of signal. Wavelet coefficients are obtained by carrying out a discrete wavelet transform.

$T(a, b)=\frac{1}{\sqrt{a}} \int_{-\infty}^{\infty} g\left(\frac{t-b}{a}\right) x(t) d t$

where transform coefficients $T(a, b)$ are computed for specific locations on the signal, $t=b$, and for periods (which are a function of $a), g$ is the analyzing wavelet function and $x(t)$ is volume FHR signal.

For instance, if we take as wavelet family the Haar wavelets we have that the Haar wavelet family $\left\{\psi_{k}^{n}(t)\right\}$ is the orthonormal basis for the $L^{2}([0,1])$ functions:

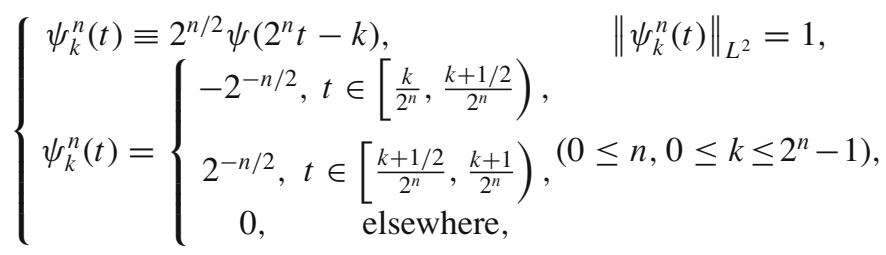

Given the discretization of the function $\left\{x_{i}\right\}, x_{i}=$ $x\left(t_{i}\right), t_{i}=i /(N-1),(i=0, \ldots, N-1), N=2^{M}$, the Lagrange polynomial interpolation of $\left\{x_{i}\right\}$ gives:

$x(t)=\sum_{i=0}^{N-1} l_{i}(t) x_{i}, \quad l_{i}(t) \equiv \prod_{k=0, \ldots, i-1, i+1, \ldots, N-1} \frac{\left(t-t_{k}\right)}{\left(t_{i}-t_{k}\right)}$,

being $l_{i}(t)$ the Lagrange coefficients.

With the Haar wavelets and the discretization: $a=$ $2^{-n}, b=2^{-n} k$ the continuous wavelet transform becomes

$$
\begin{gathered}
T\left(2^{-n}, 2^{-n} k\right)=-2^{-n} \sum_{i=0}^{N-1} x_{i}\left[\int_{k / 2^{n}}^{(k+1 / 2) 2^{n}} \prod_{k=0, \ldots, i-1, t+1, \ldots, N-1}\right. \\
\left.\times \frac{\left(t-t_{k}\right)}{\left(t_{i}-t_{k}\right)} d t-\int_{(k+1 / 2) / 2^{n}}^{k / 2^{n}} \frac{\left(t-t_{k}\right)}{\left(t_{i}-t_{k}\right)} d t\right],
\end{gathered}
$$

The wavelet transform allows to decompose a signal into components, in our case, we consider only 8 frequency bands (Figs. 1 and 2). Thus we could choose the appropriate frequency scale from 8 ones in the wavelet transform and analyze some amplitude characteristics (maximum range (MR), standard deviation (SD) and mean absolute deviation (MAD)) while ignoring the contribution of other frequency scales.

A comparison between the groups are performed by using Student $t$-test for continuous variables with normal distribution and Mann-Whitney $U$-test for continuous variables without normal distribution. The values with the probability less than 0.05 are considered statistically inessential. Multiple logistic regression was used to investigate the diagnostic significant of FHR gain-frequency characteristics and to create diagnostic model to predict anterpartum asphyxia. Diagnostic model is created to analyze data of training sample of CTGs. 40 CTGs without accelerations, decelerations, bradyand tachycardia, with mean oscillation amplitude ranged from 3 to 5 beat $/ \mathrm{min}$ were included in training sample. 20 CTGs of training sample were obtained from women with abnormal perinatal outcome and 20 CTGs of training sample were obtained from women with normal perinatal outcome. Efficiency of the created diagnostic model is checked by analyzing data of examination sample of the rest 81 CTGs.

\section{Results}

FHR gain-frequency characteristics of training sample of CTGs are analyzed in order to find new diagnostic criteria of fetal condition. There were no statistical significant differences between all amplitude characteristics in five low frequency bands excepting MR ranged from 0.01 to $0.02 \mathrm{~Hz}$ and from 0.04 to $0.07 \mathrm{~Hz}$. SD and MAD of FHR in three high frequency bands $(>0.3 ; 0.14-0.3 ; 0.07-0.14 \mathrm{~Hz})$ are statistically significant lower in cases of intrauterine deaths. The area under the ROC curve and its SE is greatest for MAD in frequency bands from $0.14-0.3 \mathrm{~Hz}(0.83,0.06, p<0.001, n=$ 40). These data allow to predict intrauterine death by analysis of CTGs without accelerations, decelerations, brady- and tachycardia, with mean oscillation amplitude from 3 to 5 beat/min (Tables 1 and 2). Multiple logistic regression was used to investigate the relationship of FHR gain-frequency characteristics and anterpartum fetal asphyxia and to create diagnostic model to predict perinatal outcome. Variables were selected by a forward-step procedure. The criterion for selection in the model was probability value of $<0.05$. The diagnostic model included $\mathrm{SD}$ in range $>0.3 \mathrm{~Hz}$ and MAD ranged from 0.14 to $0.3 \mathrm{~Hz}$. The created diagnostic model is detecting compromise fetal condition in 18 from 20 CTGs (90\%, $\left.\mathrm{CI}_{95 \%}=68-99 \%\right)$ obtained in cases of intrauterine deaths and normal fetal condition in 18 from 20 CTGs obtained in cases of normal perinatal outcome. Diagnostic efficacy for CTGs training sample was $90 \%\left(\mathrm{CI}_{95 \%}=76-\right.$ 97\%). We have checked diagnostic efficiency of the created model by analyzing the rest 81 CTGs from the examination sample whose data are not analyzed at the creating diagnostic model. It is used to determine the compromised fetal conditions in 17 from 17 CTGs obtained in cases of intrauterine deaths and normal fetal condition in 58 cases from 64 CTGs $\left(91 \%, \mathrm{CI}_{95 \%}=81-96 \%\right)$ obtained in cases of normal perinatal outcome. The comparison of the diagnostic efficiency of training and examination samples of CTGs confirms high sensitivity and specification of our model. 
Fig. 2. The wavelet decomposition of a fetal heart rate in 8 frequency bands

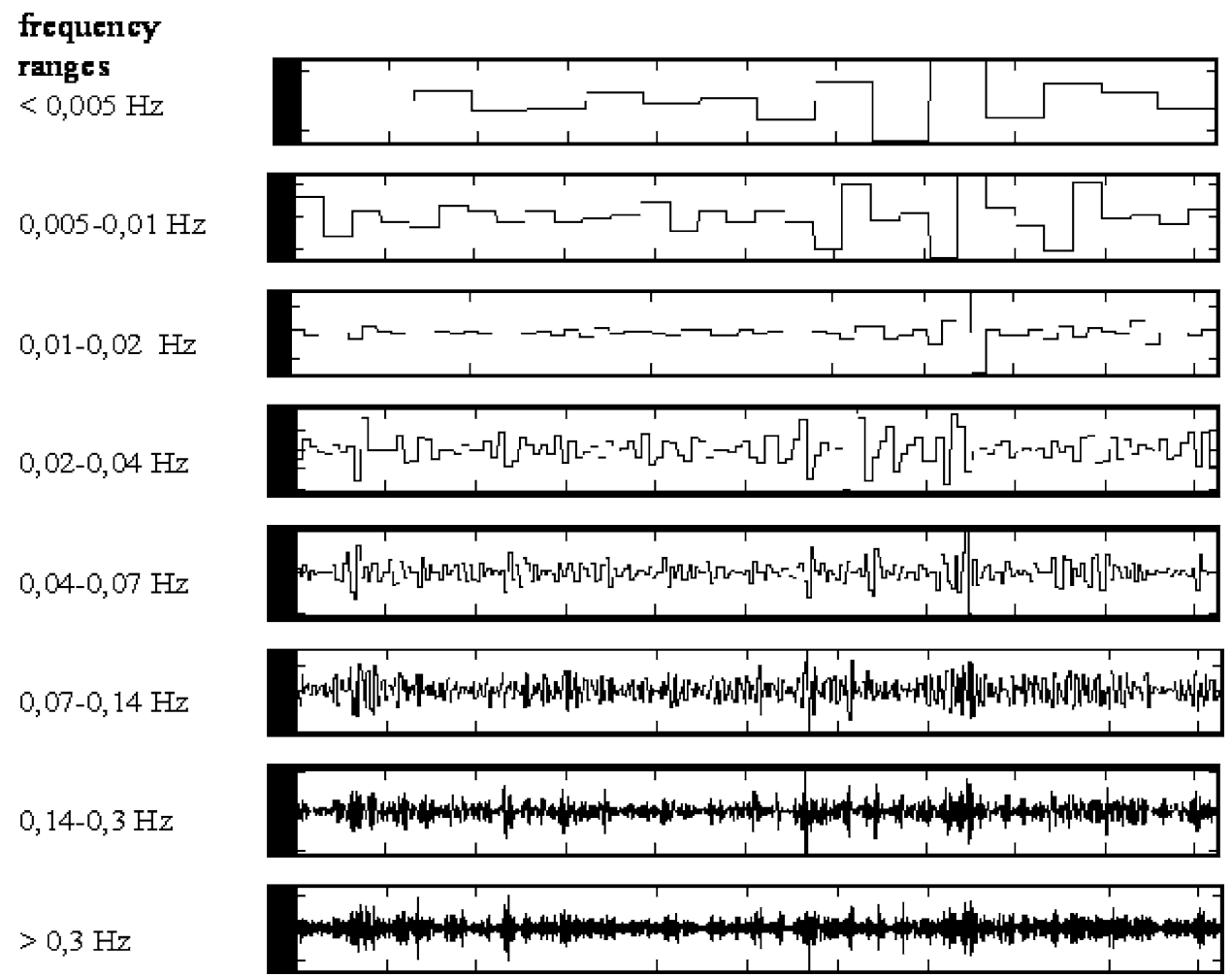

We perform certain additional computations.

1. Median volume ratio $\mathrm{SD}$ to $\mathrm{MAD}$ in frequency band $>0.3 \mathrm{~Hz}$ is statistically essentially higher (1.34, quartile boundaries from 1.28 to $1.43, n=20$ ) in cases of intrauterine deaths than in cases of normal perinatal outcome (1.28, quartile boundaries from 1.22 to $1.32, n=20$, $p=0.01)$. The area under the ROC curve and its SE for volume ratio $\mathrm{SD}$ to $\mathrm{MAD}$ in frequency range $>0.3 \mathrm{~Hz}$ to predict intrauterine death was $0.73(\mathrm{SE}=0.06, p=0.012$, $n=40)$.

2. The areas under the ROC curves and their SE for the difference between volume SD in frequency ranges from 0.14 to $0.3 \mathrm{~Hz}$ and volume SD in frequency range $>0.3 \mathrm{~Hz}$ and the difference between volumes MAD of the same frequency range was $0.76(\mathrm{SE}=0.07, p=0.003, n=40)$ and $0.84(\mathrm{SE}=0.06, p<0.001, n=40)$, respectively.

Table 1 FHR gain-frequency characteristics of training sample of CTGs (high frequency bands)

\begin{tabular}{|c|c|c|c|c|c|c|c|c|c|c|c|c|}
\hline \multirow[b]{2}{*}{ CTGs sample } & \multicolumn{4}{|c|}{ MR (beats/min) } & \multicolumn{4}{|c|}{$\mathrm{SD}$ (beats/min) } & \multicolumn{4}{|c|}{ MAD (beats/min) } \\
\hline & $M$ & CI $95 \%$ & $\mathrm{Me} / \mathrm{N}$ & QI & $M$ & CI $95 \%$ & $\mathrm{Me} / \mathrm{N}$ & QI & $M$ & CI $95 \%$ & $\mathrm{Me} / \mathrm{N}$ & QI \\
\hline \multicolumn{13}{|c|}{ Frequency range $>0.3 \mathrm{~Hz}$} \\
\hline$I^{*}$ & 4.6 & $3.2-6.1$ & $3.7 / 0.001$ & $2.9-4.8$ & 0.32 & $0.28-0.37$ & $0.3 / 0.1$ & $0.26-0.36$ & 0.23 & $0.21-0.26$ & $0.23 / 0.4$ & $0.2-0.26$ \\
\hline $\mathrm{II}^{*}$ & 4.5 & $3.7-5.3$ & $4.3 / 0.08$ & $2.9-5.4$ & 0.38 & $0.34-0.43$ & $0.38 / 0.04$ & $0.31-0.48$ & 0.3 & $0.27-0.33$ & $0.28 / 0.08$ & $0.25-0.37$ \\
\hline$p^{* *}$ & & & 0.5 & & & & 0.049 & & & & 0.01 & \\
\hline \multicolumn{13}{|c|}{ Frequency range $0.15-0.3 \mathrm{~Hz}$} \\
\hline $\mathrm{I}$ & 6.1 & $4.7-7.6$ & $5.4-0.01$ & $4.5-6.6$ & 0.54 & $0.48-0.6$ & $0.56 / 0.5$ & $0.41-0.62$ & 0.39 & $0.36-0.43$ & $0.39 / 0.6$ & $0.34-0.43$ \\
\hline II & 6.8 & $5.9-7.7$ & $6.9 / 0.5$ & $5.1-8.1$ & 0.67 & $0.6-0.75$ & $0.65 / 0.09$ & $0.54-0.8$ & 0.52 & $0.46-0.58$ & $0.49 / 0.06$ & $0.42-0.62$ \\
\hline$p$ & & & 0.13 & & & & 0.02 & & & & 0.0004 & \\
\hline \multicolumn{13}{|c|}{ Frequency range $0.07-0.15 \mathrm{~Hz}$} \\
\hline I & 7.6 & $5.9-9.4$ & $7.0 / 0.01$ & $4.6-9.2$ & 0.87 & $0.77-0.98$ & $0.87 / 0.8$ & $0.69-1.02$ & 0.63 & $0.57-0.7$ & $0.64 / 0.8$ & $0.52-0.74$ \\
\hline II & 8.7 & $7.5-9.8$ & $8.1 / 0.8$ & $7.2-10.0$ & 1.03 & $0.93-1.13$ & $1.03 / 0.08$ & $0.83-1.24$ & 0.77 & $0.7-0.84$ & 0.76 & $0.65-0.91$ \\
\hline$p$ & & & 0.09 & & & & 0.03 & & & & 0.006 & \\
\hline \multicolumn{13}{|c|}{ Frequency range $0.04-0.07 \mathrm{~Hz}$} \\
\hline I & 8.2 & $6.8-9.5$ & $7.7 / 0.001$ & $6.6-8.4$ & 1.2 & $1.1-1.3$ & $1.2 / 0.9$ & $0.96-1.35$ & 0.86 & $0.78-0.94$ & $0.88 / 0.6$ & $0.71-0.98$ \\
\hline II & 11.8 & $9.3-14.4$ & $10.2 / 0.001$ & $8.4-13.9$ & 1.4 & $1.2-1.6$ & $1.4 / 0.6$ & $1.1-1.6$ & 0.98 & $0.87-1.1$ & $0.98 / 0.2$ & $0.74-1.17$ \\
\hline$p$ & & & 0.01 & & & & 0.06 & & & & 0.1 & \\
\hline
\end{tabular}


Table 2 FHR gain-frequency characteristics of training sample of CTGs (low frequency bands)

\begin{tabular}{|c|c|c|c|c|c|c|c|c|c|c|c|c|}
\hline \multirow[b]{2}{*}{ CTGs sample } & \multicolumn{4}{|c|}{ MR (beats/min) } & \multicolumn{4}{|c|}{$\mathrm{SD}$ (beats/min) } & \multicolumn{4}{|c|}{ MAD (beats/min) } \\
\hline & $\bar{M}$ & CI 95\% & $\mathrm{Me} / \mathrm{N}$ & QI & $\bar{M}$ & CI 95\% & $\mathrm{Me} / \mathrm{N}$ & QI & $\bar{M}$ & CI $95 \%$ & $\mathrm{Me} / \mathrm{N}$ & QI \\
\hline \multicolumn{13}{|c|}{ Frequency range $0.02-0.04 \mathrm{~Hz}$} \\
\hline I & 9.1 & $7.3-10.9$ & $8.1 / 0.3$ & $6.1-11.8$ & 1.4 & $1.2-1.6$ & $1.3 / 0.3$ & $1.1-1.7$ & 1.1 & $0.9-1.2$ & $0.9 / 0.1$ & $0.9-1.2$ \\
\hline II & 11.5 & $9.1-13.9$ & $10.6 / 0.3$ & $7.7-15.1$ & 1.5 & $1.3-1.8$ & $1.5 / 0.5$ & $1.1-1.9$ & 1.1 & $0.9-1.2$ & $1.0 / 0.5$ & $0.8-1.4$ \\
\hline$p$ & & & 0.1 & & & & 0.5 & & & & 0.9 & \\
\hline \multicolumn{13}{|c|}{ Frequency range $0.01-0.02 \mathrm{~Hz}$} \\
\hline I & 6.7 & $5.5-7.9$ & $6.2 / 0.4$ & $5.3-8.9$ & 1.4 & $1.2-1.6$ & $1.5 / 0.9$ & $1.1-1.7$ & 1.1 & $0.9-1.3$ & $1.1 / 0.8$ & $0.8-1.3$ \\
\hline II & 11.3 & $8.3-14.3$ & $8.0 / 0.01$ & $7.5-16.6$ & 1.7 & $1.4-2.1$ & $1.6 / 0.06$ & $1.4-1.9$ & 1.1 & $1.0-1.3$ & $1.1 / 0.5$ & $0.8-1.4$ \\
\hline$p$ & & & 0.01 & & & & 0.2 & & & & 0.9 & \\
\hline \multicolumn{13}{|c|}{ Frequency range $0.005-0.01 \mathrm{~Hz}$} \\
\hline I & 6.2 & $4.9-7.6$ & $6.1 / 0.08$ & $3.8-8.0$ & 1.4 & $1.2-1.7$ & $1.4 / 0.2$ & $1.0-1.7$ & 1.1 & $0.9-1.3$ & $1.1 / 0.4$ & $0.8-1.3$ \\
\hline II & 6.9 & $5.0-8.9$ & $5.6 / 0.001$ & $4.5-7.7$ & 1.5 & $1.2-1.8$ & $1.4 / 0.01$ & $1.0-1.7$ & 1.1 & $0.9-1.3$ & $1.1 / 0.03$ & $0.8-1.3$ \\
\hline$p$ & & & 0.8 & & & & 1.0 & & & & 0.8 & \\
\hline \multicolumn{13}{|c|}{ Frequency range $<0.005 \mathrm{~Hz}$} \\
\hline I & 5.1 & $4.1-6.2$ & $4.6 / 0.01$ & $3.4-5.9$ & 1.4 & $1.2-1.7$ & $1.3 / 0.7$ & $1.1-1.8$ & 1.2 & $1.0-1.4$ & $1.1 / 0.5$ & $1.0-1.5$ \\
\hline II & 5.4 & $4.0-6.8$ & $4.5 / 0.01$ & $3.5-6.3$ & 1.4 & $1.1-1.7$ & $1.2 / 0.001$ & $1.0-1.6$ & 1.1 & $0.9-1.3$ & $1.0 / 0.04$ & $0.9-1.4$ \\
\hline$p$ & & & 0.9 & & & & 0.5 & & & & 0.4 & \\
\hline
\end{tabular}

Note. I - The training sample CTGs in the cases of intrauternine deaths $(n=20)$; II - The training sample CTGs in the cases of normal perinatal outcome $(n=20)$; N - result of Shapiro-Wilk test: “+” - normal distribution, "-" - abnormal distribution; CI 95\% - 95\% confidence limits for means; QI - quartile limits for medians; $p$ - a probability value obtained by a comparison between the group means was performed with Student $t$ tests fpr variables with normal distribution (and results Levene equal-varince test) and with Mean-Whitney $U$ tests for variables with abnormal distribution.

\section{Discussion}

We have applied wavelet analysis in order to find new useful criteria to predict perinatal outcome. There exists no computer program to detect fetal condition by FHR analysis using any special mathematical approach (fast Fourier transform, wavelet transform) in clinical practice. Efficiency for detecting fetal conditions on the second stage of labour is shown only for $\mathrm{SD}$ in the scale corresponding to the central frequencies $0.04 \mathrm{~Hz}$.

The most effective criteria of computerized FHR pattern is STV. The volume of this criterion is influenced both by $0.26 \mathrm{~Hz}$ oscillations and oscillations of all other frequencies. To exclude the effect of STV volume at the decelerations periods containing them we discard these time-intervals from analysis. However STV cannot distinguish the low FHR variation of healthy fetuses during quiet sleep from the low variation of compromised fetuses.

Our results show that the most effective of the analyzed FHR gain-frequency characteristics is MAD in the frequency bands from 0.14 to $0.3 \mathrm{~Hz}$. We conclude that this criteria is close to STV but it gives a new method which allows to minimize the influence of all other frequencies. On the base of an analysis of CTGs without accelerations, decelerations, brady- and tachycardia, with mean oscillation amplitude from 3 to 5 beats/min discover that it is effective in prediction of the intrauterine death. In particular it gives pos- sibility to perform an analysis of low variable CTGs which give no information for visual analysis.

We show that the study of different amplitude characteristics - SD and MAD - and amplitude characteristics of different frequencies allows to increase the accuracy of the perinatal outcome prediction. We find out that changing volume between different amplitude characteristics and between amplitude characteristics of different frequency ranges has a diagnostic significance. Indeed ratio SD to MAD in frequency range $>0.3 \mathrm{~Hz}$ was statistic significant lower in cases of normal perinatal outcome. It can reflect on the increasing of fluctuation variability FHR in frequency bands $>0.3 \mathrm{~Hz}$ in the case of compromise fetuses. Both the quotient between volume SD in frequency range from 0.14 to $0.3 \mathrm{~Hz}$ and volume SD in frequency range $>0.3 \mathrm{~Hz}$ and the quotient between volumes MAD of the same frequency range were statistic significant lower in cases of intrauterine death. It can reflect on the decreasing of the fluctuation variability FHR between amplitude characteristics of different high frequency bands for compromise fetuses.

Acknowledgements The work by second, third and fifth authors is partially supported by the Belarusian Fund for Fundamental Scientific Research. The work of CC was partially supported by Regione Campania under contract: "Modelli nonlineari di materiali compositi per applicazioni di nanotecnologia chimica-biomedica". LR 28/5/02 n. 5, Finanziamenti 2003. This article is a part of a joint Programme on mathematical modeling and applications developed in cooperation 
of scientists from Belarusian State University (Minsk, Belarus) and University of Salerno (Salerno, Italy).

\section{References}

1. van Geijn, H. P., Cardiotocography, Textbook of Perinatal Medicine (Editor-in-Chief Asim Kurjak), Parthenon Publishing 1998.

2. Borgotta, L., Shrout, P. E., and Divon, M. Y., Reliability and reproducibility of nonstress test readings. Am. J. Obstet. Gynecol. 159:554-558, 1988.

3. Mantel, R., van Geijn, H. P., Ververs, I. A., and Copray, F. J., Automated analysis of near-term ante-partum fetal heart rate in relation to fetal behavioral states: the Sonicaid System 8000. Am.J. Obstet. Gynecol. 165:57-65, 1991.

4. Dildy, G. A., Clark, S. L., and Loucks, C. A., Intrapartum fetal pulse oximetry: Past, present, and future. Am.J. Obstet. Gynecol. 175:1-9, 1996.
5. Pardey, J., Moulden, M., and Redman, C. W. G., A computer system for the numerical analysis of nonstress tests. Am. J. Obstet. Gynecol. 186:1095-1103, 2002.

6. Pumprla, J., Howorka, K., Groves, D., Chester, M., and Nolan, J., Functional assessment of heart rate variability: physiological basis and practical applications. Int. J. Cardiol. 84:1-14, 2002.

7. Addison, P. S., The Illustrated Wavelet Transform Handbook. Bristol: IoP Publ., 2002.

8. Salamalekis, E., Thomopoulos, P., Giannaris, D., Salloum, I., Vasios, G., Prentza, A., Koutsouris, D., Computerized intrapartum diagnosis of fetal hypoxia based on fetal heart rate monitoring and fetal pulse oximetry recordings utilizing wavelet analysis and neural networks. Br. J. Obstet. Gynaecol. 109:1137-1142, 2002.

9. Zelianko, E. N., Voskresenskij, S. L., Kirilenko, V. P., and Doubrovina, O. V., An evolution in approaches to cardiotocographic estimate of fetal wellbeing. Medical Panorama (9):12-17, 2004 (in Russian). 